\title{
A!
}

This is an electronic reprint of the original article.

This reprint may differ from the original in pagination and typographic detail.

Kuznetsov, A.; Cuesta, F. S.; Ptitcyn, G. A.; Wang, X.; Tretyakov, S. A.

\section{Coherent Asymmetric Absorber}

Published in:

2021 15th International Congress on Artificial Materials for Novel Wave Phenomena, Metamaterials 2021

DOI:

10.1109/Metamaterials52332.2021.9577097

Published: 20/09/2021

Document Version

Peer reviewed version

Please cite the original version:

Kuznetsov, A., Cuesta, F. S., Ptitcyn, G. A., Wang, X., \& Tretyakov, S. A. (2021). Coherent Asymmetric Absorber. In 2021 15th International Congress on Artificial Materials for Novel Wave Phenomena, Metamaterials 2021 (pp. 105-107). IEEE. https://doi.org/10.1109/Metamaterials52332.2021.9577097

This material is protected by copyright and other intellectual property rights, and duplication or sale of all or part of any of the repository collections is not permitted, except that material may be duplicated by you for your research use or educational purposes in electronic or print form. You must obtain permission for any other use. Electronic or print copies may not be offered, whether for sale or otherwise to anyone who is not an authorised user. 


\title{
Coherent Asymmetric Absorber
}

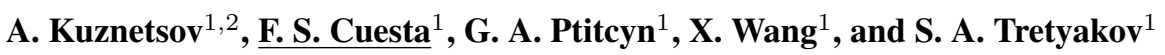 \\ ${ }^{1}$ Department of Electronics and Nanoengineering, School of Electrical Engineering, Aalto University, \\ P.O. Box 15500, FI-00076 Aalto, Finland \\ ${ }^{2}$ School of Electronic Engineering, Moscow Institute of Electronics and Mathematics, HSE University, \\ Tallinskaya Ulitsa, 34, 123458, Moscow, Russia \\ francisco.cuestasoto@aalto.fi
}

\begin{abstract}
Coherent illumination allows additional flexibility over metasurface functionalities, with coherent perfect absorbers as one typical example. However, most of coherent metasurface devices are based on spatially uniform metasurfaces that offer a limited control over scattered waves. In this talk, we present an idea of coherent asymmetric absorbers with periodic surface impedances. The proposed metasurface can function as a perfect retroreflector for coherent incidences from one side and as a perfect absorber for incidences from the other side.
\end{abstract}

\section{INTRODUCTION}

Metasurfaces allow control of tangential electric and magnetic fields by creating a discontinuity in space. From the variety of inclusion topologies, electric-only polarizable metasurfaces are easy to design and fabricate, yet they are quite versatile. However, these metasurfaces do not create discontinuities of the tangential electric field, reducing possible functionalities. As an example, a metasurface absorber in form of a lossy sheet can only absorb up to $50 \%$ of the incident power since the tangential electric field is continuos across the sheet. Nevertheless, coherent illumination of such sheets helps to reach $100 \%$ absorption [1-6]. However, physically, coherent perfect absorbers do not show additional advantages beyond their intended purpose of reducing reflections. On the other hand, inhomogeneous metasurfaces bring more flexible applications through control of non-specular reflection and transmission [7]. As an example, a coherent retroreflector has been proved to be useful to prevent the propagation of a single mode through a parallel-plate waveguide [8].

In this talk, we explore new capabilities of inhomogeneous coherent metasurfaces, focusing on the dependence of structures' absorption at different incident angles, controlled by the phase difference between coherent incident waves. This kind of metasurfaces can lead to developments of thin sensitive sensors capable to distinguish small changes of the incident angle, amplitude, and phase.

\section{COHERENT ABSORPTION}

Let us consider a metasurface as a boundary between two homogeneous space regions, as shown in Fig. 1. This metasurface can be represented by an electric surface current density, proportional to the tangential electric field at the boundary. This approach gives us significant design freedom, as long as the net tangential electric field remains continuous at the both sides of the metasurface. For certain applications, like total absorption, illuminating the structure from one side is not enough, and coherent illumination (coming from a mirror or a secondary source) is required. An inhomogeneous surface impedance profile can bring additional scattering properties to the structure, since more than one Floquet mode can propagate in space.

Let us assume that a set of two coherent waves $\left(E_{I 2}=E_{I 1} \exp \left[j \phi_{I 2}\right]\right)$ illuminates both sides of the metasurface from an oblique direction $\theta_{i}$ (see Fig. 1). We design the metasurface as a retroreflector that reflects the incident waves back to the sources with the reflection amplitude $\Gamma$ and phase $\phi_{R}$, respectively. Knowing the incident and reflected fields at both sides of the metasurface, the surface impedance of the structure can be uniquely determined, for TE and TM waves respectively, as

$$
Z_{s}^{\mathrm{TE}}=\frac{\eta_{0}}{2 \cos \theta_{i}} \frac{\exp \left[-2 j k y \sin \theta_{i}\right]-\Gamma \exp \left[j \phi_{R}\right]}{\exp \left[-2 j k y \sin \theta_{i}\right]+\Gamma \exp \left[j \phi_{R}\right]},
$$


(a)

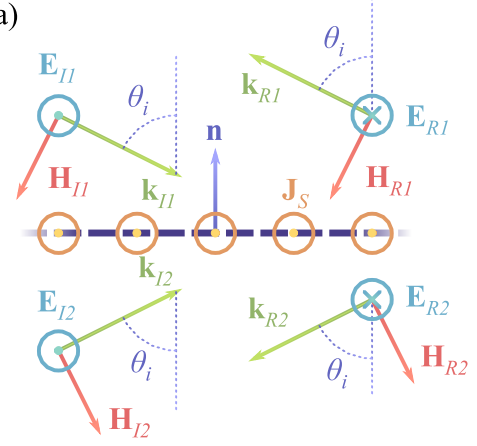

(b)

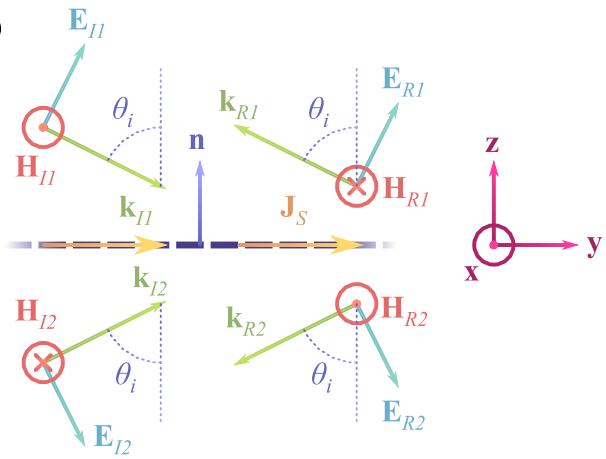

Fig. 1: Incident and reflected plane waves from coherent retroreflective electromagnetic metasurfaces which are designed for (a)-transverse electric and (b)-transverse magnetic polarizations.

$$
Z_{s}^{\mathrm{TM}}=\frac{\eta_{0} \cos \theta_{i}}{2} \frac{\exp \left[-2 j k y \sin \theta_{i}\right]+\Gamma \exp \left[j \phi_{R}\right]}{\exp \left[-2 j k y \sin \theta_{i}\right]-\Gamma \exp \left[j \phi_{R}\right]} .
$$

Please notice that known perfect coherent retroreflector and perfect coherent absorber are extreme scenarios, that correspond to $\Gamma=1$ and $\Gamma=0$, respectively.

As soon as the surface impedance is determined, we can analyze the reflecting properties for coherent incidences from other illumination angles. The total reflection for coherent incidences is decomposed into different modes, which can be analyzed using the mode-matching method developed in [9]. The absorption of the structure is found by subtracting the power reflected into free space from the total incident power.
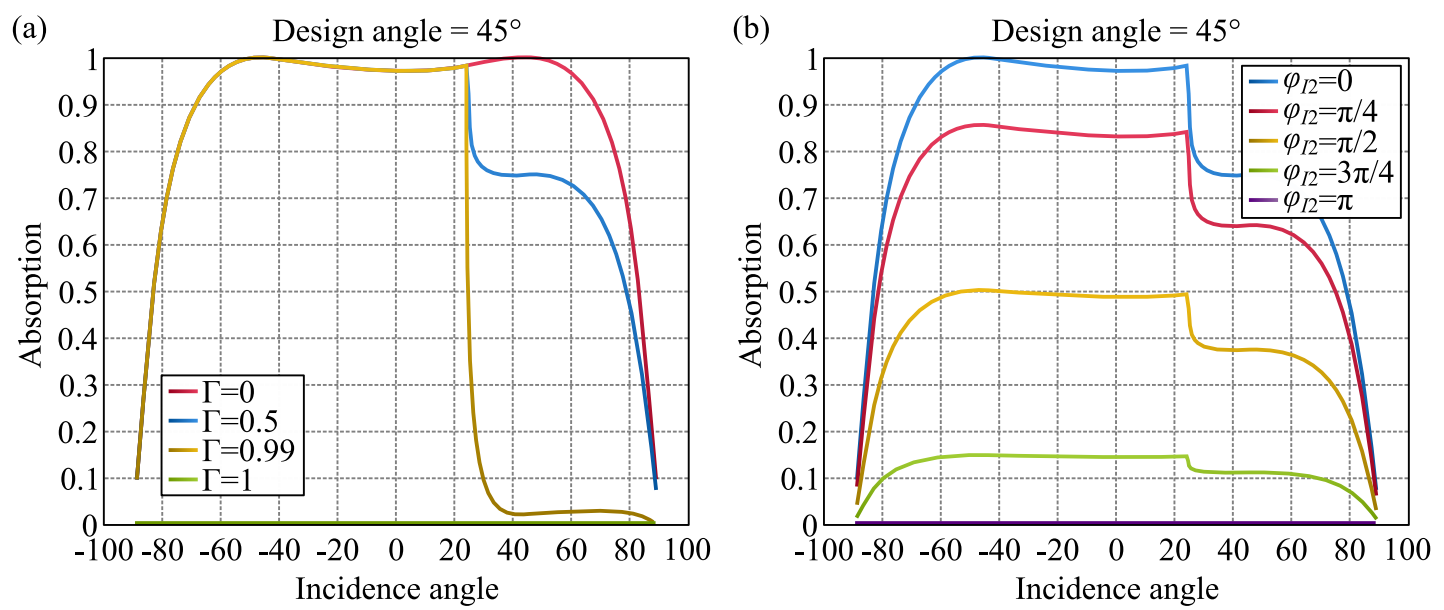

Fig. 2: Absorption obtained from a coherent retroreflective absorber for different angles of incidence, considering two scenarios: (a) Using surface impedances with different reflection coefficients $\Gamma$ at $\theta_{i}=45^{\circ}$, and (b) Using a coherent source with the phase difference between top and bottom illumination $\phi_{I 2}$ in a structure designed for $\Gamma=0.5$ at $\theta_{i}=45^{\circ}$.

Results shown in Fig. 2 have been achieved by calculating the absorption for designs with different reflection coefficients $\Gamma$ or different phase differences between the top and bottom illuminations $\phi_{I 2}$, assuming the design incident angle as $\theta_{i}=45^{\circ}$. These results are potentially attractive for sensing applications, as a retroreflective absorber designed for $\Gamma \approx 1$ presents a sharp slope near its operational conditions, ideal for angle detection, as shown in Fig. 2(a). On the other hand, results in Fig. 2(b) become essential for phase detection, as the absorption depends strongly on the phase difference between the top and bottom illuminations. 


\section{CONCLUSIONS}

Here, we have introduced and discussed the concept of coherent retroreflective absorbers. The properties of this structure appears promising for sensing applications, where sharp angle and phase variations are essential for applications related with source stability in laser generation.

\section{REFERENCES}

[1] G. Nie, Q. Shi, Z. Zhu, and J. Shi, "Selective coherent perfect absorption in metamaterials," Applied Physics Letters, vol. 105, no. 20, p. 201909, 2014.

[2] D. G. Baranov, A. Krasnok, T. Shegai, A. Alù, and Y. Chong, "Coherent perfect absorbers: linear control of light with light," Nature Reviews Materials, vol. 2, no. 12, oct 2017.

[3] W. Lv, J. Bing, Y. Deng, D. Duan, Z. Zhu, Y. Li, C. Guan, and J. Shi, "Polarization-controlled multifrequency coherent perfect absorption in stereometamaterials," Opt. Express, vol. 26, no. 13, pp. 17236-17 244, Jun 2018.

[4] X. Feng, J. Zou, W. Xu, Z. Zhu, X. Yuan, J. Zhang, and S. Qin, "Coherent perfect absorption and asymmetric interferometric light-light control in graphene with resonant dielectric nanostructures," Opt. Express, vol. 26, no. 22, pp. $29183-29191$, Oct 2018.

[5] J. Zou, F. Xiong, J. Zhang, and Z. Zhu, "Coherent perfect absorption in artificially engineered nanometer metal/semiconductor composite films at oblique incidence," OSA Continuum, vol. 2, no. 11, pp. 3251-3261, Nov 2019.

[6] Z. Zhang, X. Zhang, Y. Xu, X. Chen, X. Feng, M. Liu, Q. Xu, M. Kang, J. Han, and W. Zhang, "Coherent chiralselective absorption and wavefront manipulation in single-layer metasurfaces," Advanced Optical Materials, vol. 9, no. 3, p. 2001620, 2021.

[7] X. Wang, A. Díaz-Rubio, V. S. Asadchy, G. Ptitcyn, A. A. Generalov, J. Ala-Laurinaho, and S. A. Tretyakov, "Extreme asymmetry in metasurfaces via evanescent fields engineering: Angular-asymmetric absorption," Phys. Rev. Lett., vol. 121, p. 256802, Dec 2018.

[8] F. S. Cuesta, G. A. Ptitcyn, M. S. Mirmoosa, and S. A. Tretyakov, "Coherent retroreflective metasurfaces," 2020, arXiv:2012.14391.

[9] X. Wang, A. Díaz-Rubio, and S. A. Tretyakov, "Independent control of multiple channels in metasurface devices," Phys. Rev. Applied, vol. 14, p. 024089, Aug 2020. 